\title{
MULTISPECTRAL AND MULTISCALE INFRARED IMAGING FOR THE STUDY OF HEAT AND MASS TRANSFER IN HETEROGENEOUS MEDIA
}

\author{
C. Pradere ${ }^{1}$, A. Sommier ${ }^{1}$, S. Chevalier ${ }^{1}$, E. Abisset ${ }^{1}$ and J.C. Batsale ${ }^{1}$ \\ ${ }^{l}$ I2M, UMR CNRS 5295, Esplanade des arts et Métiers 33405 Talence Cedex, France.
}

In the first part, the study of multiscale heterogeneous solid materials has been extended to the study of multispectral heat and mass transfer in microfluidic systems. In fact, the recent development of the Fast Infrared Imaging Spectroscopic Technique (FIIST) [1] allows simultaneous acquisition of temperature and concentration fields, using a non-intrusive method and also Thermal Imaging Velocimetry (TIV) [2]. In this work, the exothermic acid-base reaction between sodium hydroxide and hydrochloric acid is performed in a coflow microfluidic chip of high aspect ratio. This configuration makes it possible to obtain laminar flows where the mixture of the species is then only due to a radial diffusion process and advection. Mass diffusion cone makes out since water transmittance is increased in presence of the ion pair of $\mathrm{Na}^{+}$and $\mathrm{Cl}^{-}$. Both heat and mass diffusivities are estimated from the logarithmic parabolas method [3], originally developed for thermal transfer but applied here also in the mass transfer case since the diffusion transport equation which is similar in both cases.

Finally, in a last part, the beginning of thermospectroscopic tomography will be presented with imaging system that is able to measure transient temperature phenomena taking place inside a bulk by $3 \mathrm{D}$ tomography. This novel technique combines the power of multispectral waves and the high sensitivity of infrared imaging. The tomography reconstruction is achieved by the 3D motion of the sample at several angular positions followed by inverse Radon transform processing to retrieve the 3D transient temperatures. The aim of this novel volumetric imaging technique is to locate defects within the whole target body as well as to measure the temperature in the whole volume of the target. This new-fashioned thermal tomography will open research perspectives in the non-invasive monitoring techniques for volume inspection and in-situ properties estimations.

[1] Romano, M., C. Ndiaye, A. Duphil, A. Sommier, J. Morikawa, J. Mascetti, J.C. Batsale, L. Servant, et C. Pradere. «Fast Infrared Imaging Spectroscopy Technique (FIIST) ». Infrared Physics \& Technology 68 (2015): $152-58$.

[2] C. Pradere, M. Joanicot, J.C. Batsale, J. Toutain and C. Gourdon, (2006), "Processing of temperature field in chemical microreactors with infrared thermography", Quantitative InfraRed Thermography Journal, ISSN: 1768-6733, vol 3, nº 1 , pp 117-135.

[3] Gaverina, L., J. C. Batsale, A. Sommier, et C. Pradere. « Pulsed Flying Spot with the Logarithmic Parabolas Method for the Estimation of In-Plane Thermal Diffusivity Fields on Heterogeneous and Anisotropic Materials ». Journal of Applied Physics 121, no 11 (2017): 115105. 